\title{
Solubility in fluids close to their critical points
}

\author{
Roberto Fernández-Prini \\ Departamento Química de Reactores, Comisión Nacional de Energía Atómica, \\ Av. Libertador 8250, CP-1429 and Instituto de Química Física de Materiales, \\ Ambiente y Energía, Facultad de Ciencias Exactas y Naturales, UBA, Ciudad \\ Universitaria, CP-1428, Buenos Aires, Argentina.
}

\begin{abstract}
A feature of solubility in near-critical fluids is its strong dependence on pressure and temperature. A general thermodynamic procedure which describes the distribution of solutes between two coexisting phases, may be conveniently employed to describe solubility in near-critical fluids. Asymptotic critical relations are a great help to evaluate data for volatile solutes, for solid solutes they help to separate contingent dependences of solubility from those which are essential. This analysis suggests there is no need to invoke the existence of critical clustering to explain the observed behaviour.
\end{abstract}

\section{INTRODUCTION}

In order to describe completely the gaseous and liquid states of matter, it is essential to have a knowledge of the thermodynamic region surrounding the critical point. Dilute multicomponent fluid systems in the vicinity of the solvent's critical point exhibit a behaviour which is very different from that of solutions under ordinary conditions. This peculiar behaviour, due to the superposition of the enhanced solvent susceptibility (long range effect) with the changing fluid density which affects the short range intermolecular interactions, offers a challenge to the Physical Chemistry of Solutions. Moreover, these systems are attracting an increasing interest due to the ever more frequent use of supercritical fluids as suitable reaction media for many chemical processes. Solubility and its temperature and pressure dependences are important thermodynamic quantities to characterize the intermolecular interactions responsible for the physicochemical behaviour of near-critical solutions. The experimental determination of solubilities becomes increasingly difficult when $T \rightarrow T_{c 1}$, this effect is particularly disturbing when the solubility of gases along the solvent liquid-vapour coexistence curve is being determined because at $T=T_{c 1}$ the two coexisting fluid phases become identical.

When dealing with near-critical mixtures it is necessary to establish which is the most convenient manner of describing their thermodynamic behaviour. The properties of these mixtures are dominated by density and solute partial molar quantities become inadequate to express the observed behaviour because they diverge at infinite dilution and at the solvent's critical temperature, $T_{c 1}$; a feature that has often been interpreted as the result of clustering of solvent molecules around solute particles. It is convenient to discuss solubility in near-critical fluids as a special case of the distribution or partition of substances between the solution and another phase, which may correspond to a pure solute phase, e. $g$. for solid solutes.

\section{SOLUBILITY MEASUREMENTS AND DISTRIBUTION CONSTANTS.}

For the case of volatile (gaseous) solutes, the dissolution process is usually described in terms of Henry's constant, $k_{\mathrm{H}}^{\infty}$, defined by the infinte dilution ratio of the solute fugacity, $f_{2}$, to its mole fraction, $x$, in the liquid,

$$
k_{\mathrm{H}}^{\infty}=\lim _{x \rightarrow 0} \frac{f_{2}}{x}
$$

In order to calculate $k_{\mathrm{H}}^{\infty}$ from $(p, T, x)$ it is necessary [1],[2] to use an equation of state for the gaseous mixture, to take explicit account of the pressure dependence (Poynting effect) and of the solute nonideality, these corrections[2] have increasing influence in the determination of Henry's 
constant as $T \rightarrow T_{c 1}$. To calculate them it is necessary to use an iterative semiempirical method, thus the uncertainty of $k_{\mathrm{H}}^{\infty}$ becomes quite significant already at $100 \mathrm{~K}$ from $T_{c 1}$ [3]. Figure 1 is a plot of the high temperature $\ln \left(k_{\mathrm{H}}^{\infty} / p^{\ominus}\right)$ for the systern $\mathrm{N}_{2}-\mathrm{H}_{2} \mathrm{O}$ as function of the temperature[3][4]. Fig. 1 shows that the Poynting and non-ideality corrections to $k_{\mathrm{H}}^{\infty}$, which have opposite sign, become very large with increasing temperature, so that the resulting values of $k_{\mathrm{H}}^{\infty}$ in the high temperature range become more uncertain. One last point to be noted is that the change of $\ln k_{\mathrm{H}}^{\infty}$ with temperature along the coexistence curve diverges at the critical point as illustrated in Fig. 1; thus, small temperature fluctuations will perturb significantly the equilibrium.

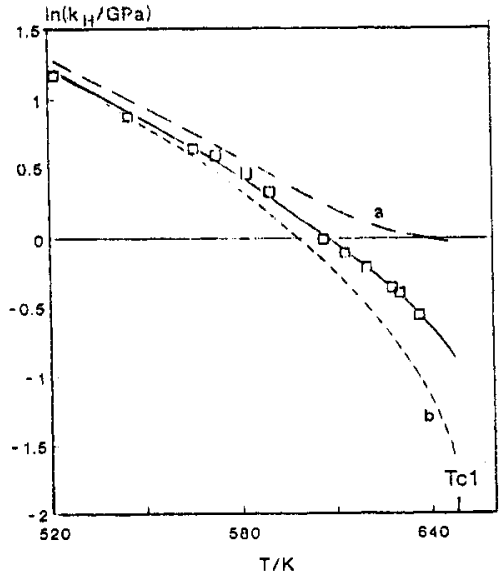

Figure 1. $\ln \left(k_{\mathrm{H}}^{\infty} / \mathrm{GPa}\right)$ against temperature for $\mathrm{N}_{2}$ in water close to $T_{c 1}[3]$. $\square$ : final values of $k_{\mathrm{H}}^{\infty}$.

Curve a: without Poynting correction. Curve b: without correction for liquid nonideality.

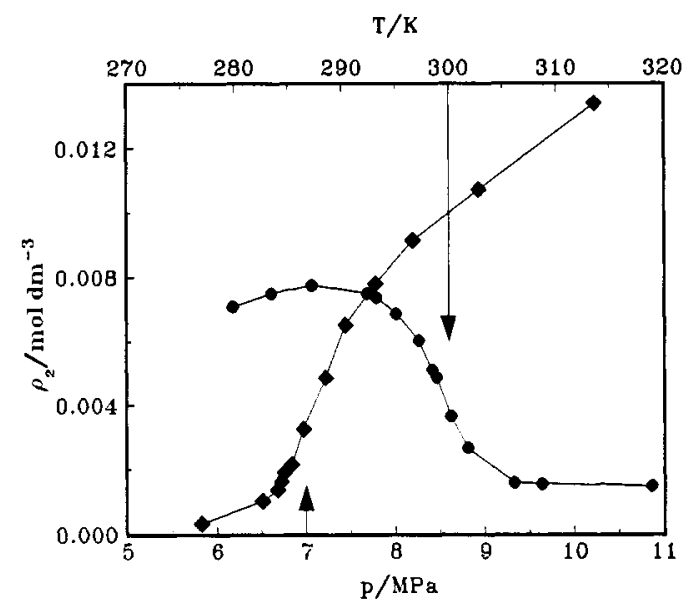

Figure 2. Isobaric and isothermal volumetric solubility $\left(\rho_{2} \equiv x \rho_{1}^{*}\right)$ of $\mathrm{I}_{2}$ in supercritical Xe.[5] $\bullet:\left(T / T_{c 1}\right)=1,031 . \bullet:\left(p / p_{c 1}\right)=1.23$.

We have studied the solubility of several polar and nonpolar solid solutes in near-critical solvents. Figure 2 shows a representative example of the isobaric and isothermal solubilities of solid $I_{2}$ in supercritical $\mathrm{Xe}$ as function of $T$ and $p$ respectively. It is quite clear that the $(p, T)$ dependence of solubility is very strong in the region where the solvent's susceptibility or expansivity pass through extrema (indicated by arrows in the Fig. 2). The consequence of this behaviour is illustrated in Figure 3, where the solute's partial molar volumes at infinite dilution obtained from the pressure dependence of solubility are plotted against solvent density for the systems $\mathrm{SF}_{6}-\mathrm{CHI}_{3}$ and $\mathrm{CHF}_{3}-$ $\mathrm{CHI}_{3}$. This behaviour is also found by direct density measurements[6]. It has been frequently observed that density is a more natural variable than pressure or temperature for fluids far from their triple points[7][8]. However for isobaric runs the density dependence of solubility still shows an inflection point. It has been suggested [9] that the isobaric solubility may be understood simply by considering that the behaviour in the low and high fluid density regions is that characteristic of gases and liquids respectively, while around the critical density the behaviour of the solution is dominated by the enhanced susceptibility of the near-critical solvent.

When considering the solubility of solid solutes it is important to divide the dissolution process in two steps. In the first step the solid is vaporized at $(T, p)$ and in the second step the vapour is dissolved in the near-critical solvent. In this way it is possible to use the same scheme to analyze the solubility of condensed pure phases and of gases. The enhancement factor defined by the ratio of the actual solubility to the ideal solubility, that is,

$$
\mathcal{E}=\frac{x}{x^{\mathrm{id}}}=\frac{x p}{p_{2}^{*}}
$$

is a convenient quantity which takes account of the second step in the dissolution of a solid. Due to the fact that the use of $\mathcal{E}$ eliminates the contribution of the increase of the solid solute vapour 
pressure with temperature, which is significant at low density, $\mathcal{E}$ is a monotonous function of the reduced density for isobaric as well as for isothermal runs [9] as illustrated below.

The concept of solubility implies that the solute in a solution is in equilibrium with that contained in another phase, this may be another mixture or a pure solute condensed phase, often a solid. Thus solubility is a particular case of the equilibrium of partition or distribution of a solute between two phases. The advantage of the generalization is that the same thermodynamic formalism (vide infra) may be used to describe both processes. The distribution constant is defined as the ratio of $y$, the concentration of solute in the second phase $\mathrm{v}$, to that in the fluid phase at infinite dilution,

$$
K_{D}=\lim _{x \rightarrow 0} \frac{y}{x}
$$

$K_{D}$ is related to the difference of standard chemical potential of the solute in the two coexisting phases,

$$
R T \ln K_{D}=\mu_{2}^{\infty}(\mathrm{f})-\mu_{2}^{\infty}(\mathrm{v})
$$

The enhancement factor for a solid solute may be expressed by the ratio

$$
\mathcal{E}=\frac{K_{D}^{\text {id }}}{K_{D}}
$$

where $K_{D}^{\text {id }}$ is the distribution constant of the solute in the ideal gas state. For gaseous solutes, Henry's constant is related to $K_{D}$ by,[2]

$$
k_{\mathrm{H}}^{\infty}=K_{D} \Phi_{2}^{\infty} p_{1}^{*}
$$

where $\Phi_{2}^{\infty}$ is the solute's fugacity coefficient at infinite dilution.

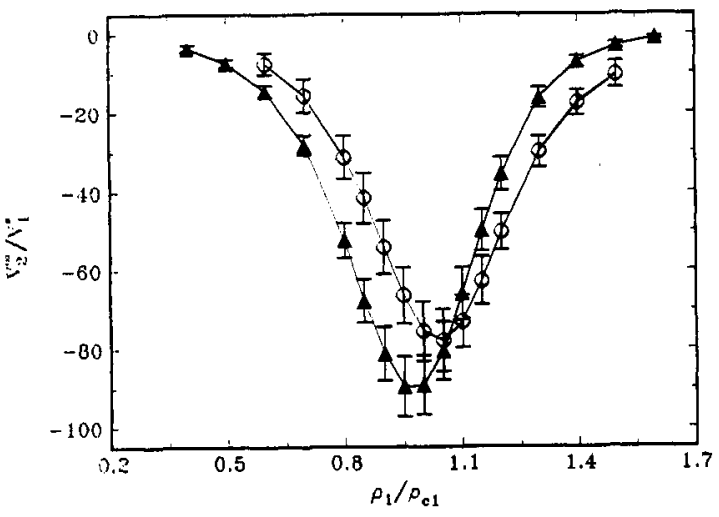

Figure 3. $\left(V_{2}^{\infty} / V_{1}^{*}\right)$ against $\left(\rho_{1}^{*} / \rho_{c 1}\right)$ for $\left(T / T_{c 1}\right)=1.01_{4}$. $\odot: \mathrm{CHI}_{3}$ in $\mathrm{CHF}_{3}[10] . \boldsymbol{\Delta}: \mathrm{CHI}_{3}$ in $\mathrm{SF}_{6}[11]$. The vertical bars indicate the uncertainty of the calculated points.

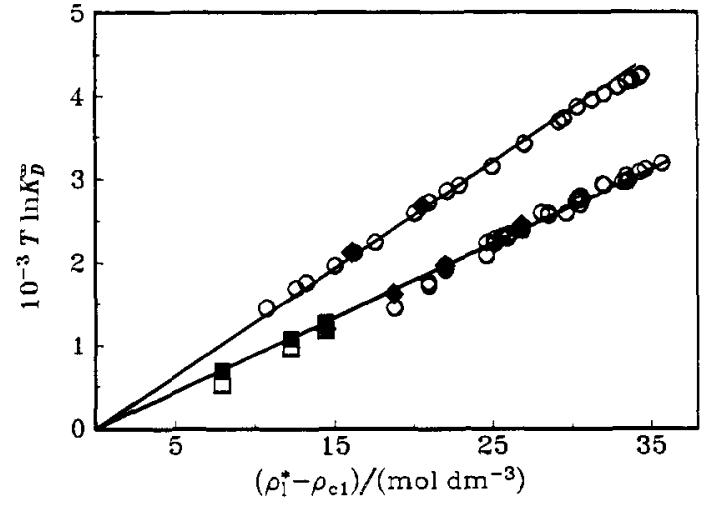

Figure 4. $T \ln K_{D}$ against $\left(\rho_{1}^{*}-\rho_{c 1}\right)$. Upper line, $\mathrm{N}_{2}-\mathrm{H}_{2} \mathrm{O}$ system; lower line $\mathrm{CO}_{2}-\mathrm{H}_{2} \mathrm{O}$ system. $\mathrm{O}$ : from gas solubility in ref [3] and [2]; : from eq(8) using data in ref [3] and [13]; ㅁ: from gas solubility data in ref [12]; : same as previous with nonideality correction (cf. text).

\section{THERMODYNAMIC DESCRIPTION OF PARTITIONING AND ASYMPTOTIC CRITICAL BEHAVIOUR.}

The necessity of employing an iterative procedure to determine $k_{\mathrm{H}}^{\infty}$ close to $T_{c 1}$, which requires the use of a model, moved us to look for an alternative procedure to describe the solubility of gases in liquids which could lend support to the results obtained with the iterative procedure. The use of the alternative procedure described in this work, together with the knowledge of the asymptotic critical behaviour of the system, has proved a poweful procedure. We have extended the use of the 
asymptotic relationships to evaluate the solubility of solids in near-critical fluids, it enables a better choice of thermodynamic variables avoiding the use of quantities having strong $(p, T)$ dependences. It is usual to start the thermodynamic analysis of phase equilibria in multicomponent mixtures with the condition that the chemical potentials of every species is the same in all the coexisting phases. The expressions resulting from this procedure are usually cumbersome when the systems are close to a point where two fluid phases become identical[14], e.g. azeotropes and critical points. A very convenient and, in many cases, powerful route to the distribution of solutes between two fluid phases, is obtained from the Gibbs-Konowalow relationships[15],

$$
[V(\mathrm{~g})-V(\mathrm{l})] d p-[S(\mathrm{~g})-S(\mathrm{l})] d T+(x-y)\left(d \mu_{2}-d \mu_{1}\right)=0
$$

where the subscripts 1 and 2 denote solvent and solute respectively. Writing explicitly $d \mu_{i}$ in terms of $(T, p, x)$ and using the Gibbs-Duhem relation, it is possible to obtain the ratio of equilibrium concentrations at constant temperature in terms of $(\partial p / \partial x)_{T, \sigma}$,

$$
\frac{x}{y}=1+\frac{(1-y)\left[V_{1}(\mathrm{~g})-V_{1}(\mathrm{l})\right]+y\left[V_{2}(\mathrm{~g})-V_{2}(\mathrm{l})\right]}{R T}\left(\frac{\partial p}{\partial x}\right)_{T, \sigma}\left(\frac{1}{1-x}-\frac{\partial \ln \gamma_{1}}{\partial x}\right)^{-1}
$$

At infinite dilution eq (7) becomes,

$$
K_{D}=1+\frac{V_{1}(\mathrm{~g})-V_{1}(\mathrm{l})}{R T}\left(\frac{\partial p}{\partial x}\right)_{T, \sigma}^{\infty}
$$

The very simple equation (8) which has the correct limit when $T \rightarrow T_{c 1}$, allows calculating $K_{D}$ from the change of total pressure with concentration in the liquid phase under conditions of coexistence of both fluid phases. When the solute is not volatile it is better to calculate the change of pressure with $y$; thus, an expression may be obtained from eq (8) exchanging the two coexisting fluid phases.

Levelt Sengers[16] has shown the convenience of deriving the asymptotic critical behaviour from the classical Taylor expansion of the Helmholtz energy, $A$, and its derivatives, in this way no strong divergence of the first derivatives are observed. Thus, starting from, [9]

$$
\mu_{2}(T, p, x)=\mu_{1}(T, p, x)+A_{x}
$$

we get,

$$
\mu_{2}^{\infty}(T, p)=\mu_{1}^{*}+A_{x}^{c}+A_{x V}^{c} \delta V+A_{x T}^{c} \delta T+\cdots
$$

the $*$ superindex denotes pure substance and the subscripts indicate the variables of differentiation. When the path is isothermal or corresponds to the coexistence of two phases, the leading term in the expansion is the $\delta V$ term and an asymptotic linear dependence of $\mu_{2}^{\infty}$ on solvent volume or density results,

$$
\mu_{2}^{\infty}(T, p) \approx \mu_{2}^{\infty, c}-\frac{A_{x V}^{c}}{\rho_{c 1}^{2}} \delta \rho
$$

Using this type of equations Japas and Levelt Sengers[17] derived the asymptotic expressions for Herny's constant,

$$
R T \ln \left(\frac{k_{\mathrm{H}}^{\infty}}{f_{1}^{*}}\right)=A+B\left(\rho_{1}^{*}(1)-\rho_{\mathrm{cl}}\right)
$$

and for $K_{D}$,

$$
R T \ln K_{D}=B\left(\rho_{1}^{*}(\mathrm{l})-\rho_{1}^{*}(\mathrm{~g})\right)
$$

Considering the law of rectilinear diameters which is obeyed close to the solvent's critical point,[18]

$$
R T \ln K_{D}=2 B\left(\rho_{1}^{*}(\mathrm{l})-\rho_{c 1}\right)
$$

The $B$ coefficient in eq (12) and (14) is related to the critical slope of the pressure as function of composition[15],

$$
B=\frac{1}{\rho_{c 1}^{2}}\left(\frac{\partial p}{\partial x}\right)_{T, V}^{\infty, c}
$$




\section{ANALYSIS OF SOME BINARY SYSTEMS}

i) Distribution of volatile solutes The $k_{\mathrm{H}}^{\infty}$ values for $\mathrm{N}_{2}-\mathrm{H}_{2} \mathrm{O}$ were determined by means of the iterative procedure starting from the values $(p, T, x)$ and using $V_{2}^{\infty}$ and $\gamma_{2}^{\mathrm{H}}$ to correct for Poynting effect and solute nonideality, these two quantities were calculated with a hard-sphere perturbation method[2][3]. It was also possible to calculate $K_{D}$ with eq (8) using the $582 \mathrm{~K}$ and $612 \mathrm{~K}$ isotherms to obtain $(\partial p / \partial x)_{T, \sigma}^{\infty}$. Figure 4 shows the close agreement between the results of this calculation and the values of $K_{D}$ calculated from solubilities using the iterative procedure[3] and eq (5). Thus the results of the iterative procedure are fully supported by the distribution constants derived from the composition dependence of the vapour pressure. Another system of great practical interest for which there was some uncertainty about the solubility at high temperature is $\mathrm{CO}_{2}-\mathrm{H}_{2} \mathrm{O}[2]$. In 1992 Crovetto and Wood[12] were able to determine $k_{\mathrm{H}}^{\infty}$ very close to the critical temperature of water. These very valuable new data when plotted together with the rest of the available solubility data as $T \ln K_{D}$ against solvent density ( $c f$. eq (14)) did not appear to extrapolate to unity at $T_{c 1}$ ( $c f$. Fig. 4). We estimated the correction of the data of Crovetto and Wood[12] due to $\mathrm{CO}_{2}$ nonideality[15] and the values of $K_{D}$ were shifted towards the expected linear behaviour indicated by eq (14). This result, which also requires the use of the iterative procedure, was verified by the calculations of the distribution constant made with eq (8) using the information about the phase diagram obtained by Takenouchi and Kennedy[13] as shown in Fig. 4.

We have recently shown that it is posssible to give formulations covering the complete temperature range of existence of liquid water, for the distribution of ten weakly interacting gaseous solutes and for $\mathrm{CO}_{2}$ between water and steam[15]. These formulations were based upon the thermodynamic procedure described above (including the asymptotic relations) and on an expression proposed by Harvey and Levelt Sengers for the low temperature region[19]. The procedure was applied only in the high temperature range for $\mathrm{HCl}, \mathrm{NH}_{3}$ and $\mathrm{H}_{2} \mathrm{~S}$ dissolved in water. In all the cases where it was possible, relationship (15) was verified within the experimental uncertainty of the involved quantities. The linear asymptotic range covered by eq (14) extends around $150 \mathrm{~K}$ from $T_{c 1}$ for the systems studied in ref([15]). This extended linear behaviour is due[9] to the great difference in volatility of the two components forming the binary system. In fact, the linear range is much smaller (only about $20 \mathrm{~K}$ ) for the system $\mathrm{H}_{2} \mathrm{O}-\mathrm{D}_{2} \mathrm{O}[14]$, supporting the contention that only when the term $A_{V x}^{c}=-(\partial p / \partial x)_{T, V}^{\infty, c}$ in eq $(10)$ is very large, the linear range extends significantly.

The generality of the alternative procedure may be judged from the fact that it has also been applied successfully to the distribution of $\mathrm{NaCl}$ and $\mathrm{KCl}$ between water and steam[15].

ii) Distribution of solid solutes For the solubility of solid solutes in near-critical fluids it is also possible to obtain an asymptotic expresion, however its use is more limited than for the case of liquid-vapour distribution because no critical point between the two phases in equilibrium exists, so that only the asymptotic behaviour of the solvent may be introduced and not the condition that both phases become identical in some thermodynamic state. The asymptotic expressions for the solubility of solids in near-critical solvents which have been explored previously by Harvey[20], are used here mainly to choose the best way to represent the data, avoiding the use of strongly varying functions and thus helping to separate contingent factors from those which are essential. Using the condition of equilibrium between the solid and the near-critical solution, the following expression may be obtained,[9]

$$
\mu_{2}^{\ominus}+R T \ln \left(\frac{f_{2}^{*}}{p^{\ominus}}\right)+\int_{p_{2}^{*}}^{p} V_{2} *(\mathrm{~s}) d p=\mu_{2}^{\infty, c}-\frac{A_{x V}^{c}}{\rho_{c 1}^{2}}\left[\rho(1)-\rho_{c 1}\right]+R T \ln \left(x \gamma_{2}\right)
$$

where $f_{2}^{*}$ is the fugacity and $V_{2}^{*}$ the molar volume of the solid solute. For slightly soluble substances deviations from nonideality may be neglected $\left(\gamma_{2} \approx 1\right)$, then the solubility is given by,

$$
\begin{aligned}
R T \ln x & =\left(\mu_{2}^{\ominus}-\mu_{2}^{\infty, c}\right)+R T \ln \left(\frac{f_{2}^{*}}{p^{\ominus}}\right)+\frac{A_{x V}^{c}}{\rho_{c 1}^{2}}\left[\rho(1)-\rho_{c 1}\right] \\
& =f(T)+\frac{A_{x V}^{c}}{\rho_{c 1}^{2}} \rho(1)
\end{aligned}
$$


where $f(T)$ denotes a slowly varying function of temperature. Using this expression together with eq (2), a simple expression for $R T \ln \mathcal{E}$ may be obtained[9]. Figures 5 and 6 show representative examples of the enhancement factors of solids dissolved in near-critical solvents; it is true to say that some systems show more curvature than those illustrated in Fig. 5 and 6 , but the majority of the systems we studied are well represented by the behaviour illustrated in the figures. It is quite notable that the curves in these figures show wide regions of linearity according to the equations derived above for the isobaric as well as for the isothermal solubilities.

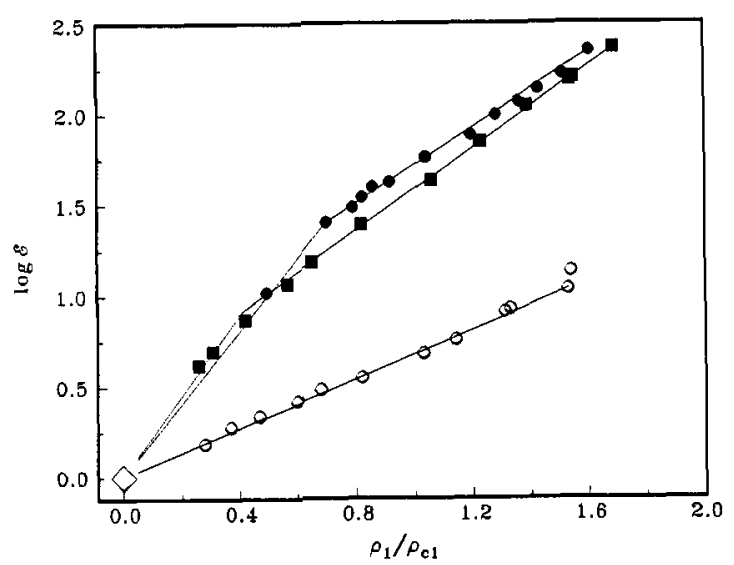

Figure 5. $\log \mathcal{E}$ against $\left(\rho_{1}^{*} / \rho_{c 1}\right)$ for isothermal runs $\left(T / T_{c 1}\right)=1.03$.

?: $\mathrm{I}_{2}$ in Xe.[5]. $\mathbf{~ : ~} \mathrm{CHI}_{3}$ in $\mathrm{CHF}_{3}[10]$. $\diamond: \mathrm{I}_{2}$ in $\mathrm{CHF}_{3}[10] . \diamond$ : origin of coordinates.

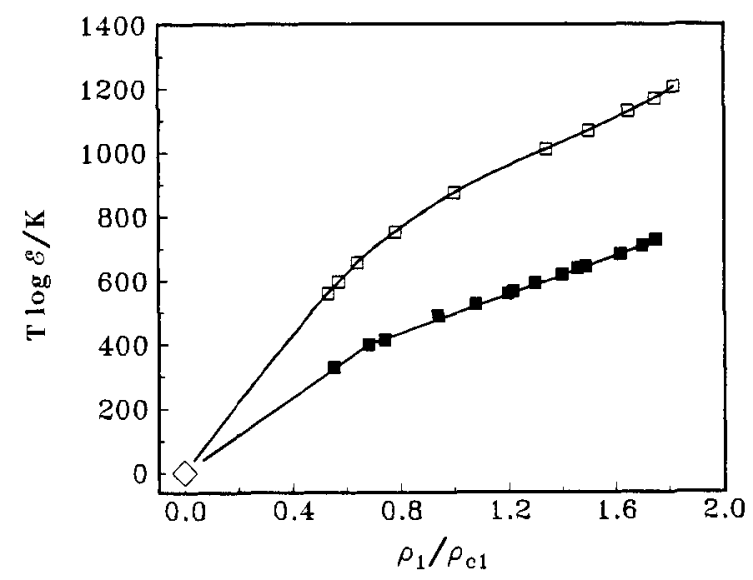

Figure 6. $\log \mathcal{E}$ against $\left(\rho_{1}^{*} / \rho_{c 1}\right)$ for isobaric runs.

a: $\mathrm{I}_{2}$ in $\mathrm{Xe},\left(p / p_{c 1}\right)=1.26 \cdot[5] \square$ : $\mathrm{CHI}_{3}$ in $\mathrm{C}_{2} \mathrm{H}_{4},\left(p / p_{c 1}\right)=1.24[11] . \diamond:$ origin of coordinates.

\section{MICROSCOPIC PICTURE}

Near-critical dilute solutions exhibit some unusual features when compared to solutions near the solvent's triple point, these include large enhancement factors and their (small) decrease with temperature, moreover differences in the solubility are emphasized in supercritical solvents when specific interactions are present[10][21]. This behaviour has stimulated speculations about the meaning of partial molar solute properties diverging to plus or minus infinity ( $c f$. Fig. 3); a dramatic change of solvent density around the solute molecules, referred to as critical clustering, has been proposed to explain it. The analysis made in the present work, shows that the behaviour observed when the fluid density varies does not require invoking a new phenomenon. It is a consequence of the coupling of the strongly enhanced solvent susceptibility in this thermodynamic region, a long range effect, with the changes in local structure around the solute molecules introduced by the varying fluid density, a short range effect. This view is supported by studies of the microscopic structure prevailing in these fluid systems[9][22]. Many thermodynamic properties are related to integrals over the volume of functions which contain the pair distribution functions, $g_{i j}(r)$ [23], so they will diverge when $g_{i j}(r)$ has a long range tail. This is the situation which is frequently interpreted as a very large excess or defect of solvent molecules around the solute particle, but really there are no evidences of critical clustering around the solutes. Figure 7 shows the total correlation function $h_{i 2}(r)=\left(g_{i 2}(r)-1\right)$ for a model Lennard-Jones mixture (values of the molecular parameters are: $\left.\left(\epsilon_{22} / \epsilon_{11}\right)=2.0,\left(\sigma_{22} / \sigma_{11}\right)=1.5,\left(k T / \epsilon_{11}\right)=1.36\right)$ according to the Percus-Yevick approximation for $\left(T / T_{c 1}\right)=1.031$ and densities which go from gas-like to liquid-like and include the critical density. It is evident that the number of first neighbours to the solute is not too much affected by the closeness of the solvent's critical point, the long range tailing-off being its most notable feature and the reason for the divergence of the integral of $h_{i 2}(r)$ taken over all the system. For the case of gases, i.e. $\rho_{1}^{*} \rightarrow 0$, the integral of $h_{i 2}(r)$ is equal to the second $(i-2)$ virial coefficient with changed sign. As the density increases, indirect interactions become more important giving rise to an incipient increase of $h_{i 2}(r)$ beyond the first peak, as shown in Fig. 7. The fact that the first peak of $h_{22}(r)$ is higher 
than that of $h_{12}(r)$ is due to the stronger solute-solute compared to the solvent-solute interactions which correspond to the case of dissolution of solids in the near-critical fluids. The inset in Fig. 7 illustrates the fact that the quantity $I(r)=r^{2} h_{12}(r)$ has a long range tail which will cause divergence of those properteis involving an integral containing $I(r)$.

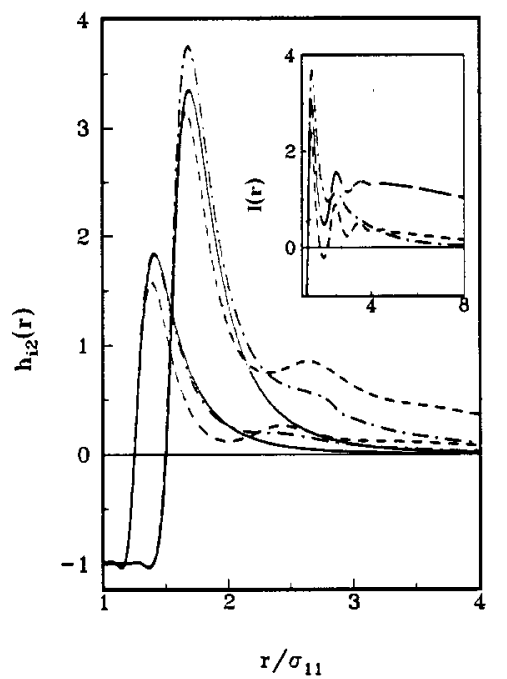

Figure 7. Total correlation functions against the distance from a solute molecule (from [9]). Lower curves, $i=1$; higher curves, $i=2 .\left(\rho_{1}^{*} / \rho_{c 1}\right)$ is $0.0-, 0.33-\cdots-\cdots, 1.02--\longrightarrow$, $1.33 \ldots$.

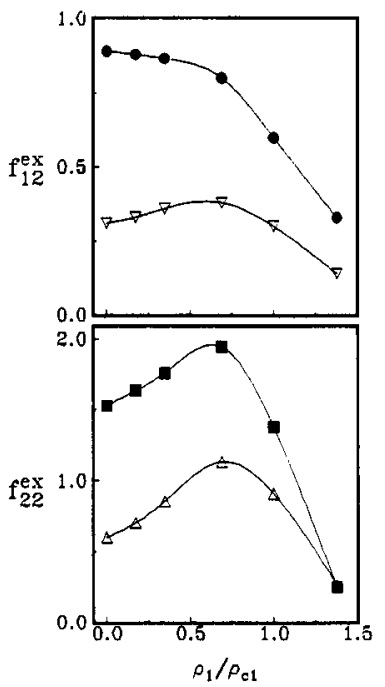

Figure 8. $f_{i 2}^{\text {ex }}$ as function of the reduced density (from ref [9]). Full symbols, first neighbours; open symbols, second neighbours.

The relative excess fraction of particles $i$ surrounding a central solute molecule, $f_{i 2}^{\mathbf{e x}}$, has been identified as a relevant variable to establish the existence of critical clustering[9][22]. Figure 8 shows the effect of solvent density upon $f_{i 2}^{\text {ex }}$ for first and second neighbours, this quantity passes through a sharper maximum for the (2-2) case than for the (1-2); the (1-2) interaction does not even produce a maximum for the excess fraction of first neighbour solvent molecules. For the excess fraction of solute, the maximum occurs at a density which is lower than the critical density, reinforcing the conclusion that effect of the short range microstructure is not coupled to the long-ranged critical fluctuations[24]. The maxima in the $f_{i 2}^{\text {ex }}$ curves are due to indirect interactions, hence they are an incipient manifestation of a characteristic feature of dense fluids. Since the solute molecules have stronger interactions with each other than with a solvent particle, at low density it would be expected that $f_{22}^{\mathrm{ex}}>f_{12}^{\mathrm{ex}}$; as the solvent density increases, its molecules will occupy positions which on the average are closer to the central solute. The solvent moiety surrounding the central solute particle provides a region where more solute or cosolvent may be preferentially attached. This may generate an incipient microheterogeneity and it could help to understand the importnat role of cosolvents in enhancing the solubility of solutes.

\section{CONCLUSIONS}

The general thermodynamic procedure presented to calculate and describe the distribution of solutes between two coexisting phases is a powerful tool which may be used over all the thermodynamic space, including near-critical regions.

The procedure takes account of the asymptotic behaviour when the two coexisting phases have a critical point.

Strong dependence of solute distribution or solubility on thermodynamic variables is avoided, expressions with smooth dependence in thermodynamic parameters are produced. 
The consequence of the analysis in the present work strongly suggest that it is unnecessary to invoke the existence of phenomena like critical clustering, this is supported by microscopic structural calculations.

\section{ACKNOWLEDGEMENT}

I am grateful to M. Laura Japas for enlightening discussions. The author is a member of Carrera del Investigador (CONICET).

\section{REFERENCE}

1. R. Fernández-Prini and R. Crovetto, AIChE J., 31, 513 (1985).

2. R. Fernández-Prini, H.R. Corti and M.L. Japas, High-Temperature Aqueous Solutions: Thermodynamic Properties, CRC Press, (1992).

3. J.L. Alvarez and R. Fernández-Prini, Fluid Phase Eq., 66, 309 (1991).

4. J.L. Alvarez, R. Crovetto and R. Fernández-Prini, Ber. Bunsenges. Phys Chem, 92, 935 (1988).

5. D. Fernández and R. Fernández-Prini, J. Chem. Thermodyn., 24, 277 (1992).

6. C.A. Eckert, D.H. Ziger, K.P. Johnston and S. Kim, J. Phys. Chem., 90, 2738 (1986).

7. E.U. Franck, Z. Physikal. Chem. N.F., 8, 192 (1956).

8. W.L. Marshall and R.E. Mesmer, J. Solut. Chem., 10, 121 (1981).

9. R. Fernández-Prini and M.L. Japas, Chem. Soc. Rev., 23, 155 (1994).

10. E. Marceca and R. Fernández-Prini, J. Chem. Thermodyn., 26, 651 (1994).

11. E. Marceca and R. Fernández-Prini, J. Chem. Thermodyn., 25, 237,719 (1993).

12. R. Crovetto and R.H. Wood, Fluid Phase Eq., 74, 271 (1992).

13. S. Takenouchi and G.C. Kennedy, Amer. J. Sci., 263, 1055 (1964).

14. M.L. Japas, R. Fernández-Prini, J. Horita and D. Wesolowski, J. Phys. Chem., submitted.

15. J.L. Alvarez, H.R. Corti, R. Fernández-Prini and M.L. Japas, Geochim. Cosmochim. Acta,, 58, 2789 (1994).

16. J.M.H. Levelt Sengers, Supercritical Fluid Technology: Reviews in Modern Theory and Applications, ed. J. Bruno and J.F. Ely, CRC Press, (1991).

17. M.L. Japas and J.M.H. Levelt Sengers, AIChE J., 35, 705 (1989).

18. J.S. Rowlinson and F.L. Swinton, iquids and Liquid Mixutures, Butterworths, (1982).

19. A.H. Harvey and J.M.H. Levelt Sengers, AIChE J., 36, 539 (1990).

20. A.H. Harvey, J. Phys. Chem., 94, 8403 (1990).

21. J.S. Murray, P. Lane, T. Brinck and P. Politzer, J. Phys. Chem., 97, 5144 (1993).

22. J.W. Tom and P.G. Debenedetti, Ind. Eng. Chem. Res., bf 32, 4573 (1993).

23. J.G. Kirkwood and F. Buff, J. Chem. Phys., 19, 744 (1951).

24. C. Carlier and T.W. Randolph, AIChE J., 39, 876 (1993). 